\title{
Is There Hope For Breast Cancer Patients Without Surgery??: An Institutional Retrospective Disease Free Survival Study.
}

\author{
Dr. Simrandeep Singh, Dr. Neeti Sharma, Dr. pramila kumari, Dr. Atul Verma, \\ Dr. Praveen Kumar, Dr. Shankar Lal Jakhar, Dr. H. S. Kumar.
}

\begin{abstract}
Background: Recently incidence of carcinoma breast has increased many folds and surpassed cervical malignancy. Management of breast carcinoma has multi modality treatment. One or the other modality, sometimes surgery is not acceptable for patients due to many reasons.

Material and method: In retrospective disease free survival study, a group of disease free patients were found who had refused for surgery and were treated by chemoradiotherapy alone.

Results: Among the 25 patients, 17 patients (68\%) were treated more than 10 years back, 5 patients (20\%) 6 years back and 3 patients (12\%) 2 years back. All these patients were disease free for above post treatment periods till april 2017.

Conclusion: Hope should not be lost for those in whom surgery is not done, this method of alone chemoradiotherapy may also yield acceptable results in terms of survival, disease free survival and cosmetic effects in some patients.
\end{abstract}

\section{Introduction}

Among women carcinoma cervix was most common malignancy, but recently incidence of carcinoma breast has increased many folds and surpassed it. Self assessment of breast cancer patients leads to presentation in very early stage. Management of breast carcinoma has multi modality treatment which involves medical, surgical and radiation oncologist. This has increased the control of this dreadful malignancy and given better survival. However patient compliance is a very important prognostic factor in determining the outcomes and survival. Sometimes multi modality treatment is not possible due to many reasons and then it becomes very difficult to overcome this malignancy. Surgery is one the method which is not accepted easily by the patients because of the invasive procedure, associated co-morbidities, fear of pain and anesthesia along with loss of breast and cosmetic effect. Sometimes patients also refuse medical and radiation treatment because of social phobia, loss of hair and stay factor. So in these settings multi modality treatment is limited. However due to early presentation in breast carcinoma, sometimes disease has very good outcome with appropriate and accurate limited treatment inspite of refusal of any treatment modality. In our institute we incidentally found some patients who were treated for breast carcinoma with medical and radiation treatment modality only due to being unfit for surgery for many reasons. On survival studies of last 15 years we found a group of patients who had no recurrence and no metastasis even though they did not opt for surgery and were treated with only chemoradiotherapy.

\section{Material and method}

On retrospective disease free survival study of breast patients, a group of disease free patients were found who had refused surgery and did not take standard multi modality treatment. All these patients were treated with only chemotherapy and radiotherapy. Tattooing was done to locate the primary tumor site accurately. 6 cycles of chemotherapy were planned for tumor regression. On clinically complete response and metastatic workup, these patients were treated with external beam radiotherapy (EBRT) and then boost to primary tumor site.

\section{Results}

Among these 25 patients, 17 patients (68\%) were treated more than 10 years back, 5 patients (20\%) 6 years back and 3 patients $(12 \%) 2$ years back. All these patients were disease free for above post treatment periods upto april 2017. 


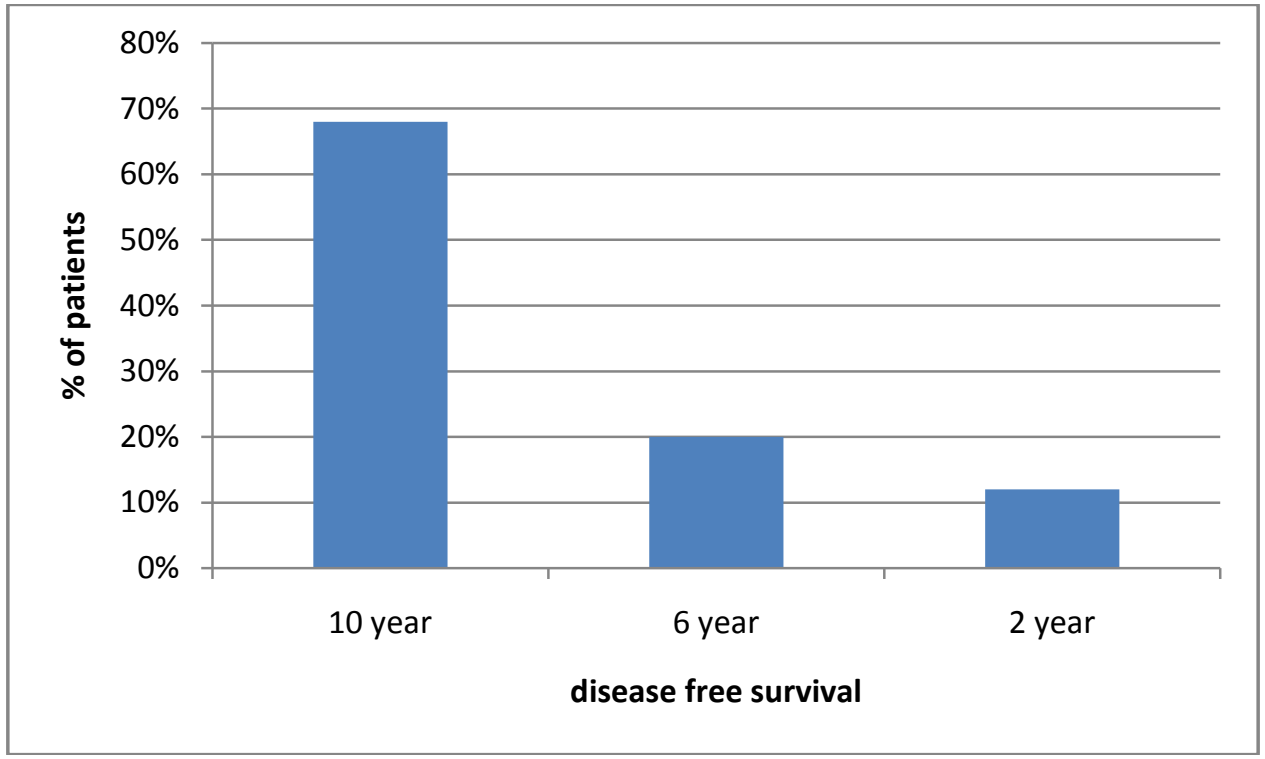

\section{Discussion}

In early stage breast cancer mastectomy or lumpectomy followed by chemoradiotherapy is offered. Many studies prove that mastectomy or lumpectomy with chemoradiotherapy have equal results in terms of overall survival and disease free survival. Fisher B and Jacobson JA have proved in their different studies that mastectomy or lumpectomy with radiotherapy have 20 years of survival in stage I and II. Angela pennisi, Laura hutchins and Monica Morrow in their different studies proved that there was pathologically complete response with neo adjuvant chemotherapy (NACT) in breast cancer. In advanced stage breast cancer mastectomy and adjuvant chemo- radiotherapy has very good outcomes and give better survival. All these studies proved that multi modality treatment is best option for breast cancer patients. During breast cancer treatment survival studies in our institute we incidentally found some patients who were treated only with medical and radiation treatment and surgery omitted due to many reasons. These patients received either adriamycin or paclitexal based chemotherapy. After the completion of 6 cycles of chemotherapy these patients were reassessed and received radiotherapy. Conventional radiotherapy was given to whole breast with total of 50Gy in 25 fractions, 2Gy per fraction, 5 fractions per week. Radiation therapy field included intact breast, chest wall and axilla with supraclavicular region. Additional boost radiotherapy was given to primary site. Out of 25 patients found without surgical management $68 \%$ had more than 10 year survival. This number may be larger as it is expected that many patients have not reported for follow up in institute on account of having no complain and being disease free. A good number may also be existing with same or other metastatic or recurrent state. However they also contribute to survival which is not mentioned in this study.

\section{Conclusion}

With multi modality treatment being the treatment of choice in breast cancer hope should not be lost for those in whom surgery is not done, this method can also give better outcome in terms of survival, disease free survival and cosmetic effects only with chemo radiation. However this incidental findings arises the need for further studies with multi institutional results and follow up to establish and maintain hope for breast cancer patients who have no courage to fight through invasive procedure.

\section{References}

[1]. Fisher B. Twenty-year follow-up of a randomized trial comparing total mastectomy, lumpectomy, andlumpectomy plus irradiation for the treatment of invasive breast cancer. New England journal of medicine.

[2]. Jacobson JA. Ten-year results of a comparison of conservation with mastectomy in the treatment of stage I and II breast cancer. New England journal of medicine.

[3]. Fisher B. Five-year results of a randomized clinical trial comparing total mastectomy and segmental mastectomy with or without radiation in the treatment of breast cancer.

[4]. Angela Pennisi, Laura Hutchins. Relevance of pathological complete response after neoadjuvant therapy for breast cancer.

[5]. Monica Morrow. Parsing pathologic complete response in patients receiving neoadjuvant chemotherapy for breast cancer. 